\title{
Study on Dynamic Disaster in Steeply Deep Rock Mass Condition in Urumchi Coalfield
}

\author{
Xing-Ping Lai, ${ }^{1,2}$ Mei-Feng Cai, ${ }^{3}$ Fen-Hua Ren, ${ }^{3}$ Peng-Fei Shan, ${ }^{1,2}$ \\ Feng Cui, ${ }^{1,2}$ and Jian-Tao Cao ${ }^{1,2}$ \\ ${ }^{1}$ School of Energy and Mining Engineering, Xi'an University of Science and Technology, Xi'an 710054, China \\ ${ }^{2}$ Key Laboratory of Western Mines and Hazard Prevention, Ministry of Education of China, Xian 710054, China \\ ${ }^{3}$ School of Civil and Environmental Engineering, University of Science and Technology Beijing, Beijing 100083, China
}

Correspondence should be addressed to Xing-Ping Lai; laixp@xust.edu.cn

Received 16 September 2014; Accepted 5 December 2014

Academic Editor: Shimin Liu

Copyright (C) 2015 Xing-Ping Lai et al. This is an open access article distributed under the Creative Commons Attribution License, which permits unrestricted use, distribution, and reproduction in any medium, provided the original work is properly cited.

The possible mining seismicity (MS) and its prediction are important for safety and recovery optimization of mining in steep-heavythick rock mass condition. The stress-lever-rotation-effect (SLRE) model of fault-like mobilization was proposed preliminarily. Some innovation monitoring technique approaches for mining seismicity assessment were successfully fulfilled at Wudong Mine of Urumchi Coalfield, China. The characteristics on acoustic-seismic-wave index indicated the spatial-temporal-strength and stress redistribution of steeply deeper-heavy thick coal and rock masses. Applications in field investigations showed that the innovation monitoring (in time and space) of these instruments could provide important information about the performance of mining disturbed structures (heading and steep pillar) during caving of competent overlying roof strata. The prediction and evaluation for mining seismicity were applicable and valid. Operating practice showed that mining efficiency was raised and conspicuous economic benefit was obtained. This approach provides essential data for assessing mining seismicity, coal burst, dynamic hazard prevention, and deep mining potential.

\section{Introduction}

There are many rock mechanics challenges related to the underground extraction of the western mining, China. Mining seismicity (MS) has a long history in Chinese coal mines [1]. Among them Urumchi Coalfield (UC) is a unique one in highly seismic region. The UC is an enrichment coalfield, as focal region of steep-heavy-thick coal seams, with the dip angle from $45^{\circ}$ to $87^{\circ}$. Its deposits are 219 hundred million tons, consisting of 30 coal seams with various thicknesses. Wudong Mine is $19.5 \mathrm{~km}$ from east to west and about $3.0 \mathrm{~km}$ from south to north. The mine area is about $38 \mathrm{~km}^{2}$. The seam thickness is varied from $30.0 \mathrm{~m}$ to $50.0 \mathrm{~m}$. The present extraction depth is around $400 \mathrm{~m}$. The dominant mining method was mechanized subhorizontal section topcoal caving (SSTCC) with annual production of 10 million tons [2]. Presently, its output is the highest among steepheavy-thick coal seams of China.
Generally, the multi-narrow-space formed above the subhorizontal section top-coal caving workings. The inclination length of working face is usually shorter. Hence, the variability of stress and deformation of steep-heavy-thick seams are obviously different from those of longwall top-coal caving (LTCC) of gently inclined coal seam [3-5]. Under the existing geological and mining conditions of the coal seam in the Dzungaria Basin, underground extraction of the coal mineral in the basin is typically accompanied by mining seismicity, which is also referred to as coal burst and dynamic collapse.

Formerly published literatures on the mining seismicity have mainly focused on understanding the mechanism of longwall top-coal caving workings involved and their impacts on the stress analysis, design and optimization, management, and policy-making [6-10]. It is novelty of the mining seismicity occurrence at Urumchi Coalfield. The field monitoring is one of crucial ways to predict mining seismicity and dynamic disaster [11]. The traditional prediction for mining seismicity 


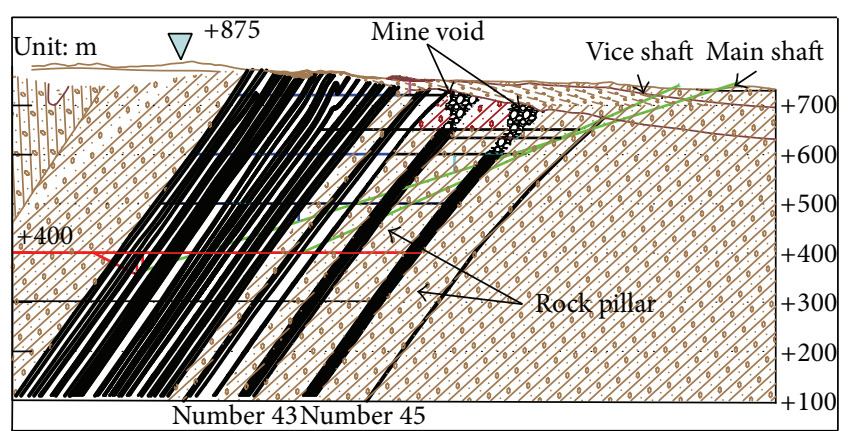

FIGURE 1: The existence of steep-heavy-thick coal seams in sectional view.

was inapplicable at Urumchi Coalfield and neither was analyzed nor studied acoustic-seismic-wave index and their implicit relations in detail. Particularly, the horizontal section coal caving multiple disturbed, the steep and broken coalrock masses caved, the size and magnitude of broken or occurrence, and intensity related to the spatial-temporalstrength relation and mining disturbance, indicating stress redistribution of coal-rock masses.

In the present study, the stress-lever-rotation-effect (SLRE) model of fault-like mobilization was preliminarily proposed and the possible mining seismicity and its prediction were performed to steeply heavy-thick coal seam and rock mass. Specifically, innovation monitoring techniques relating to the acoustic-seismic-wave index were applied at Wudong Mine, China. The approach would provide essential data for assessing mining seismicity, coal burst, dynamic hazard prevention, and deep mining potential.

\section{Site Details}

2.1. Geology and Environment. The performance of safe mining depends on the geological environment, characterizations, and geomechanical prosperities of rock masses. The Urumchi Coalfield is located in the south of the Dzungaria Basin of China and at the south edge of Urumchi City. Historically, the earthquake frequently happened here. It is an enrichment coalfield adjacent to the Western-MountainRupture Zone, which is an unwatered, ecological fragility, high ground stress region, and strong ground motion zone, known for the steep-heavy-thick coal seams. Its coal reserves are 219 hundred million tons, consisting of 30 coal seams with various thicknesses and interlayers (Figure 1). The bearing angle varies from $322^{\circ}$ to $335^{\circ}$. The roof of the rock masses is mainly the siltstone, and the fine sandstone increases at shallow and reduces at deep strata. The coal and rock masses are very rigid.

2.2. Mining Method and Condition. With the dipping angle from $45^{\circ}$ to $87^{\circ}$, the top-coal caving for steep coal seam in the horizontal section was evidently different from the gently inclined seam. Generally, the working face was arranged with short length when the thickness of coal seams ranges from $20.0 \mathrm{~m}$ to $50.0 \mathrm{~m}$. Usually, the height of the horizontal section was $20.0 \mathrm{~m}$; it would successively extend from upper place to lower sections or workings and form the typical narrowspace mining and stress concentration conditions. As shown in Figure 1, the top-coal caving workings in the horizontal section located beneath the multilayer mined-out area all the while at Wudong Mine. During the horizontal section mining disturbed under weak and laminated conditions, dynamic hazard would easily induce the relating mining seismicity, rock outburst, pillar destabilization, dynamic roof collapse, and so forth.

2.3. MS Occurrence. In general, unique mining seismicity was dominant in the experience of limited mining space. In mines, such events were often referred to as rock bursts, tremors, coal occurrence, and acoustic events, depending on their frequency contents and the sizes of the source. There was previously no mining seismicity occurrence during shallow mining, with the depth of less than $350 \mathrm{~m}$, while the coal-gas outburst ever happened. In particular, during the period of 2011-2013, the mining seismicity occurrences were severe, while mining at depths ranging from $300 \mathrm{~m}$ to $350 \mathrm{~m}$, as several hazards happened at number 43 and number 45 working faces. Figure 2 illustrated the field calamitous collapse and the coal occurrence.

\section{Proposal of the Stress-Lever-Structure Model}

The structure of mining disturbed zone is shown in Figures 3(a) and 3(b); the advance heading of number 45 working face not only undertakes the compressive and shear stress from the roof, overburden, and top-coal dynamic effect, but also resists the pry rock pillar mobilization effect of the dip of a $50.0 \mathrm{~m}$ thickness caused by number. 43 coal seam. After heading and workings being excavated, overlying gravity of excavation space is transferred into the heading on both sides, then the stress redistributes, and two peak values of $\sigma_{\max }$ present in the coal mass under the top-coal action of the roof and the floor. When the $\sigma_{\max }$ is more than the compressive strength of the coal, regional fracture and damage occurred in the coal-rock mass; meanwhile, its strength reduces to the level of residual strength. During the top-coal caving, there appears the stress-lever-rotation-effect of fault-like mobilization in the roof of number 43 workings (Figure 3(b)).

The roof-floor of shallow stope is regarded as the arch of spanning strata with a certain thickness [12]. With mining advance and disturbance, the mobilization of the roof arch and the floor is enhanced. The peak stress of the roof and the floor appears underneath the support. The $\sigma_{1 \text { max }}$, the peak stress in the coal seam, and $\sigma_{1 \max }^{\prime}$, the peak stress in the rock mass, extend downward and migrate to lower strata to form the peak stresses $\sigma_{2 \max }$ and $\sigma_{2 \max }^{\prime}$ in the lower section. The peak stress in the coal seam and the rock mass would be like this to migrate downwards successively. Mining disturbance would release stress, induce mining seismicity, or even cause dynamic hazard. It is a fundamental mechanism and a potential prediction of mining seismicity. 

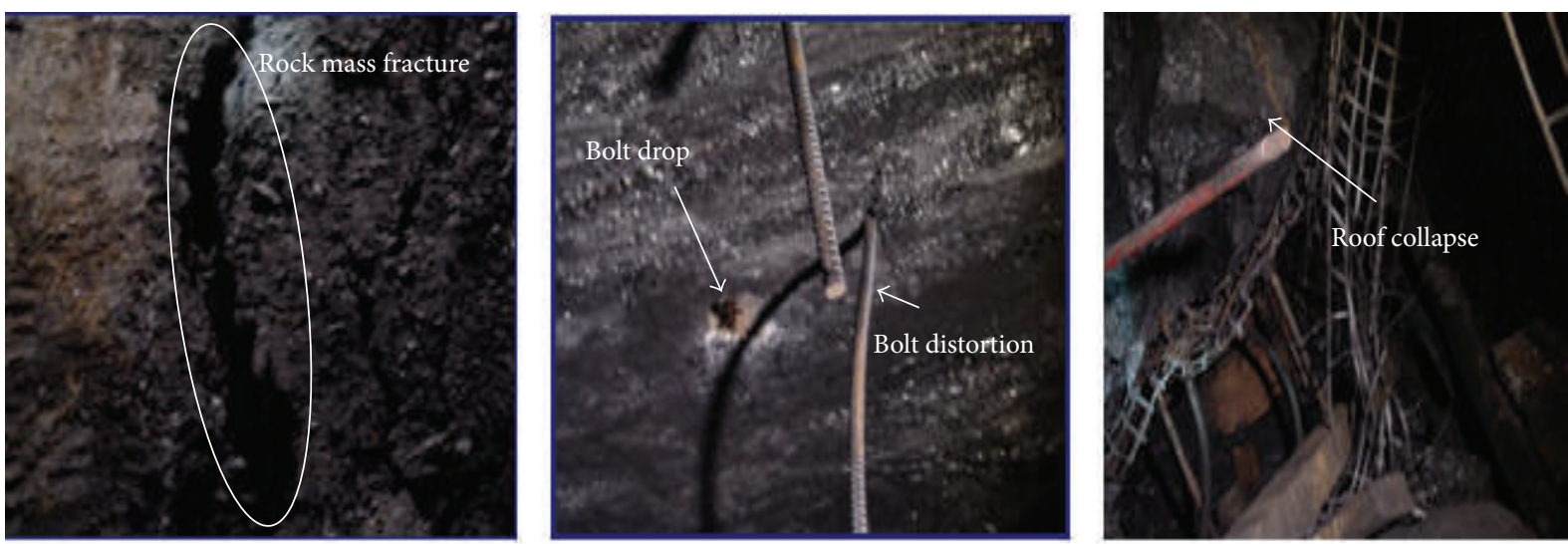

FIGURE 2: Field collapse and coal burst (2012.6.30).

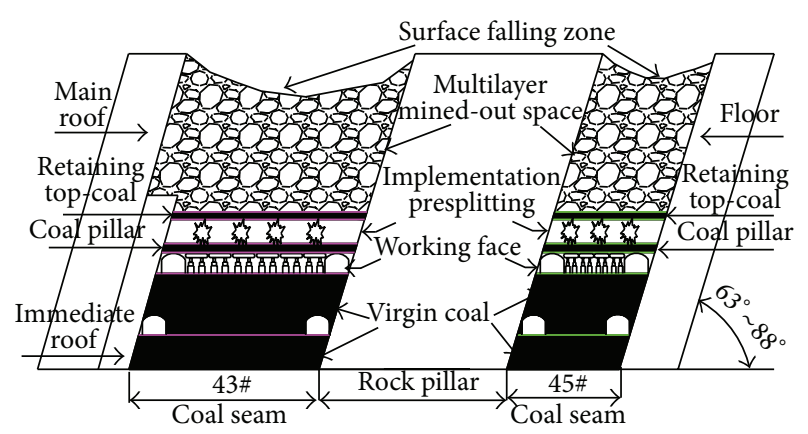

(a) Workings structure and coal-rock mass conditions

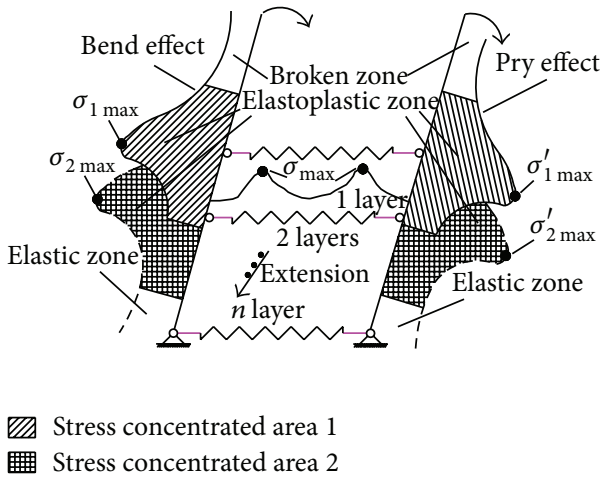

(b) Mechanical structure

FIGURE 3: Descriptions of the structure with the steep-coal-seam working face.

The stress monitoring apparatus is installed at rock pillar between number 43 and number 45 working faces. The in situ stress distribution was obtained at various drilling depths (Figure 4(a)). The numerical computation resulted from quasi-static granular displacements in block caving, arch stress, dilatancy, or swelling effects and their illustrating intrinsic evolution on shapes with the $\mathrm{FLAC}^{3 \mathrm{D}}$ program (Figure 4(b)). Also, according to multiple mining disturbed zone interaction upon block caving by means of a large 3D physical model experiment $[13,14]$, the stress distribution had two crests, and the main reason was the action of large arch of spanning strata instability caused by stress-lever-rotationeffect from the roof and the floor coal-rock mass. Multicycles of top-coal caving induced a load which was bigger than the compressive strength to the coal pillars, increasing the number and the length of the fractures.

\section{Field Acoustic-Seismic-Wave Index Monitoring}

It is vital to obtain the in situ stress distribution, deformation, and fracture characteristics upon coal-rock masses at various depths under mining disturbed conditions [1517]. The methods of acoustic-electromagnetic wave, optical imaging and ground penetrating radar (GPR), and so forth are applicable, and the comprehensive field deformation monitoring of steeply dipping coal-rock masses provides the credible and quantitative information for the assessment mining seismicity affected by redisturbance stress.

4.1. Acoustic-Wave. The quality of the surrounding rock can be determined by the value of velocity according to the principle of acoustic-wave propagation. Supposing that the coal-rock mass is elastic medium, the calculation formula of longitudinal wave is derived as follows:

$$
\nu_{P}=\sqrt{\frac{E(1-\mu)}{\rho(1+\mu)(1-2 \mu)}} .
$$

Here, $v_{P}$ is preliminary wave velocity, $E$ is elastic modulus of coal-rock mass, $\mu$ is Poisson's ratio, and $\rho$ is the density of coal-rock mass. It is indicated that the intensity and elastic modulus for the surrounding rock are smaller at the same time. Based on the elastic modulus, Poisson ratio, and other parameters gained from rock mechanics experiments of the coal-rock mass, all parameters reduced with a certain proportion for the rock mass. The longitudinal wave velocity of the coal-rock mass was $2217 \mathrm{~m} / \mathrm{s}$ and $1629 \mathrm{~m} / \mathrm{s}$ obtained by 


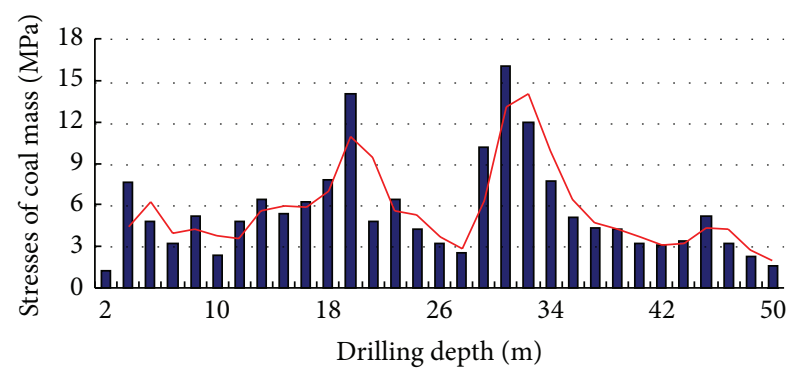

(a) Stress distribution of rock pillar at various drilling depths

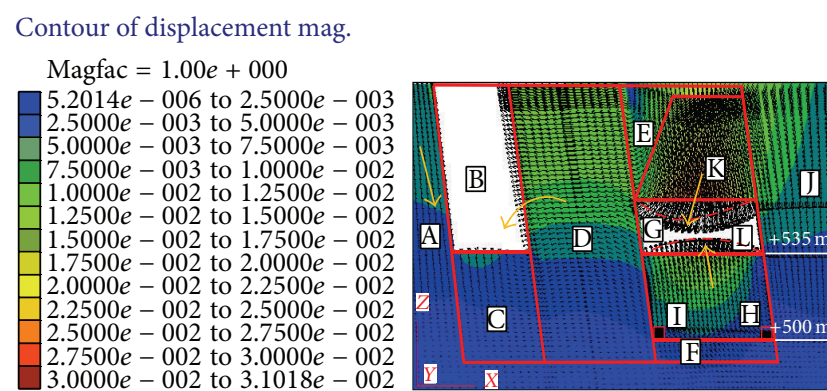
(A) Floor strata
(D) Rock pillar
(G) Caving face
(J) Roof strata
(C) $B_{1-2}$ seam
(F) $\mathrm{B}_{3-6}$ seam
(I) Tail entry
(E) Caved top-coal
(H) Head entry
(K) Top-coal flow

(b) Stress-level-rotation-effect by numerical computation

FIGURE 4: Relation between depths stress (a) and deformation distribution (b).

calculation, respectively. We proposed that the surrounding rock was fractured or ruptured when velocity of the received wave was lower than the corresponding $v_{P}$. Five test boreholes were arranged on the section of the roadway from left to right clockwise, with the angles of $0^{\circ}, 45^{\circ}, 90^{\circ}, 135^{\circ}$, and $180^{\circ}$, respectively (Figure 5). The diameter of the borehole was $0.42 \mathrm{~m}$ with the depth of $10.0 \mathrm{~m}$.

It was indicated that the velocity of longitudinal wave within the range of 0 to $1.5 \mathrm{~m}$ of coal-rock masses was less than that of analytic calculation, which means coal and rock masses qualities were poor. The acoustic velocity of the rock side was beyond that of the coal side, which showed that the stability of the rock mass side was better than that of the coal mass side. The sound velocity of measurement points was less than $2217 \mathrm{~m} / \mathrm{s}$ and $1629 \mathrm{~m} / \mathrm{s}$ in the measuring lines of $45^{\circ}$ direction for two sides and roof in the roadway with the range of $2.5 \mathrm{~m}$ to $7.5 \mathrm{~m}$. The main reason was the stress concentration effected by the nearby vertical roof bending and floor pry; the peaks $\sigma_{n \text { max }}$ and $\sigma_{n \text { max }}^{\prime}$ were produced in the two ends of the working face, respectively. The depth of the fractured zone of the roadway was about $1.5 \mathrm{~m}$, rock masses decreased within the depth of $2.5 \mathrm{~m}$ to $7.5 \mathrm{~m}$, and the stability of the borehole wall was dreadful at the $45^{\circ}$ direction for each side of the roadway. The velocity of longitudinal wave of the surrounding rock increased slightly after the distance of $7.5 \mathrm{~m}$ depth of rock masses, which indicated that the stability of roof rock masses increased. The acoustic-wave speed of the coal was less than $1629 \mathrm{~m} / \mathrm{s}$. It was indicated that the deformation and the fracture distribution were obviously asymmetric migration and localization.

4.2. Electromagnetic Wave. Delineation of mining seismicity and dynamical fractures with ground penetrating radar is an advanced way. The high frequency electromagnetic wave of ground penetrating radar launched from transmitting antenna is delivered into underground; the amplitude of electromagnetic wave changes when encountering discontinuous interface. The propagation velocity of electromagnetic wave in underground rock masses can be obtained according to the following formula:

$$
v=\frac{c}{\sqrt{\varepsilon_{r}}}
$$

Here, $c$ is $3 \times 10^{8} \mathrm{~m} / \mathrm{s}$ and $\varepsilon_{r}$ is the constant of medium. The travel time $(T)$ of the reflected wave from the target can be tracked and measured. The depth of the underground object $(H)$ can be determined based on the electromagnetic wave propagation in the medium velocity and the reflection time as follows:

$$
H=\frac{T * V}{2} .
$$

Here, $T$ is the time of electromagnetic wave propagation; $V$ is the electromagnetic wave velocity. SIR20 GPR $(100 \mathrm{MHz})$ is applied. Four lines with the $50.0 \mathrm{~m}$ length strike and that of $12.0 \mathrm{~m}$ vertical depth were detected to rock pillar at roadway.

Figure 6 illustrates the electromagnetic wave results in the roadway roof. With the frequency of 0-350 scans and the energy of 0-59 ns, the left circle in this figure shows electromagnetic wave results in a broken cavity located at the strike of 630-650 m with the vertical depth of 350-360 m, and the observed results indicate that the fragmentation degree and the electromagnetic wave energy of the coal seam are lower in the cavity; with the frequency of 350900 scans and the energy of $0-150 \mathrm{~ns}$, the right circle shows electromagnetic wave results in the other place located at the strike of 650-700 m; with the vertical depth of 360-380 m, the observed results indicate that the coal-rock masses in this place are more stable and the reflected wave has not caused any large deflections and displacements. At the strike of 700-750 m with the vertical depth of 380-400 m, wave lineups sign of delaminating and damage are observed with the electromagnetic wave results of $0-350$ scan frequency and 50-100 ns energy, which reflects obvious dislocation and mutual cross and indicates that more electromagnetic wave energy has been absorbed and the coal-rock is broken in 


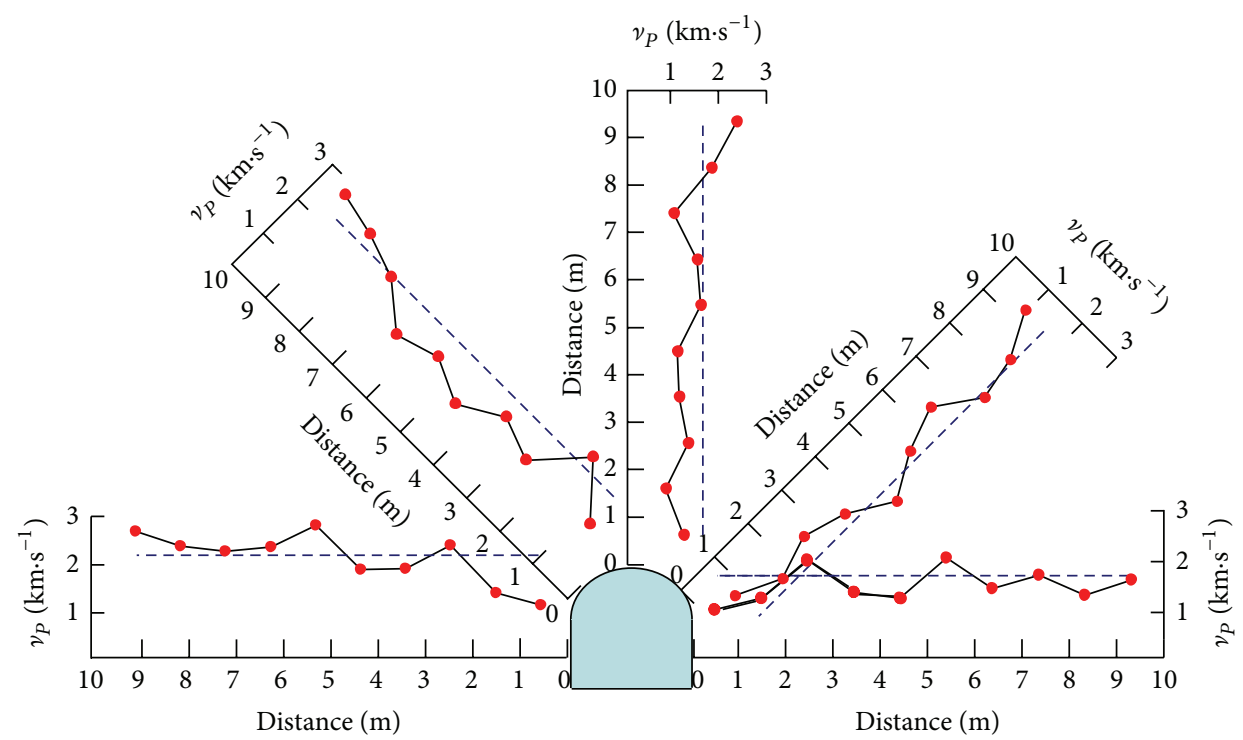

FIGURE 5: Relations of acoustic-wave velocities $\left(\nu_{P}\right)$ and the distance of boreholes.

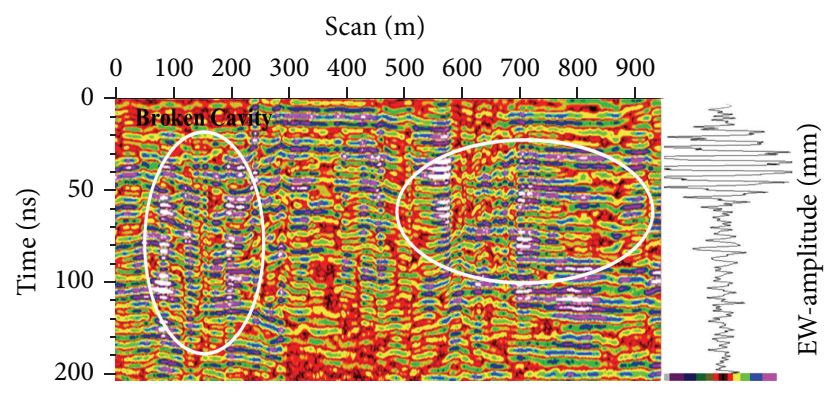

FIGURE 6: The in situ profile result of the electromagnetic wave.

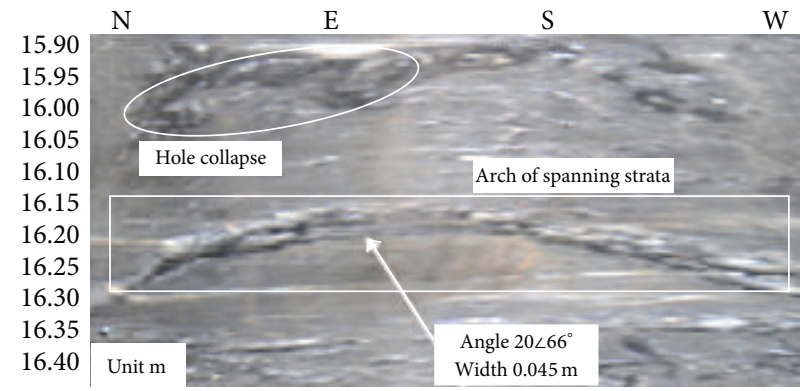

FIgURE 7: Observation by the borehole optical imaging TV.

for real-time field information. Specially, mining seismicity $\left(M_{L}=3.2\right)$ caused vast facility damage (Figure 8$)$ that happened in +500 working face (mining depth $350 \mathrm{~m}$ ) and its roadways at September 18, 2013. With analyzing the monitoring data from September 8 to September 22, 2013, amount of mining seismicity in $\mathrm{B}_{3-6}$ and $\mathrm{B}_{1-2}$ seams was 22 and 40, respectively. Energy release was more frequent. Major mining seismicity events emerged at coal mass near the rock pillar being advanced the working face. Simultaneously, mining seismicity events happened at coal mass which was lag excavation in $\mathrm{B}_{1-2}$ seams. In vertical direction, mining seismicity events mainly concentrated from $+475 \mathrm{~m}$ to $+510 \mathrm{~m}$, while major mining seismicity seldom emerged above $+550 \mathrm{~m}$. Figure 9 illustrated that mining seismicity is focused from $+500 \mathrm{~m}$ to $+510 \mathrm{~m}$ and from $+475 \mathrm{~m}$ to $+485 \mathrm{~m}$.

\section{Results and Discussions}

5.1. Mining Depth. The mining seismicity is akin to an artificial earthquake caused by mining disturbance. Mining seismicity was more frequent when the mining depth exceeds 


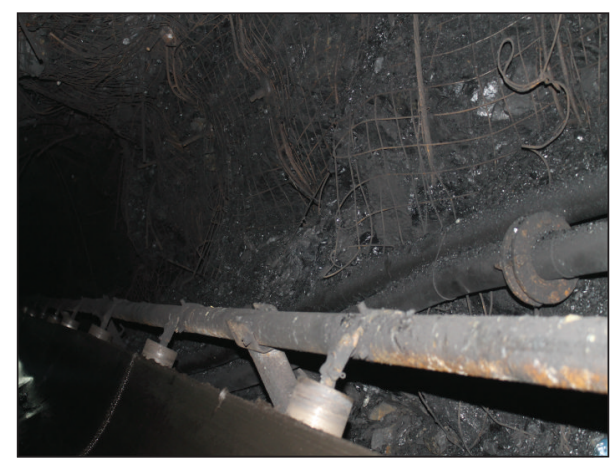

(a) Side overturn of belt conveyor

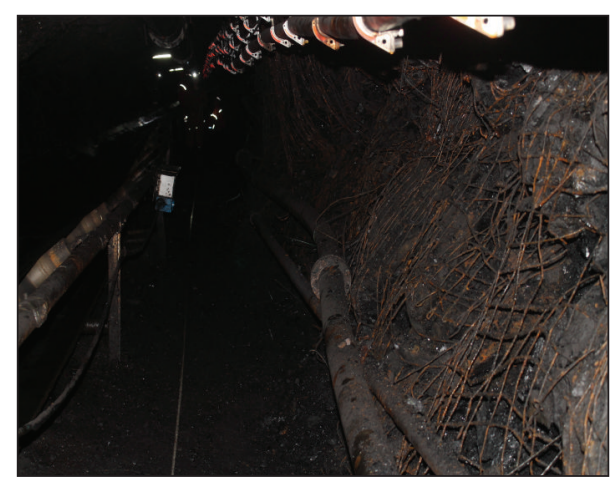

(c) Base bulge of the roadway

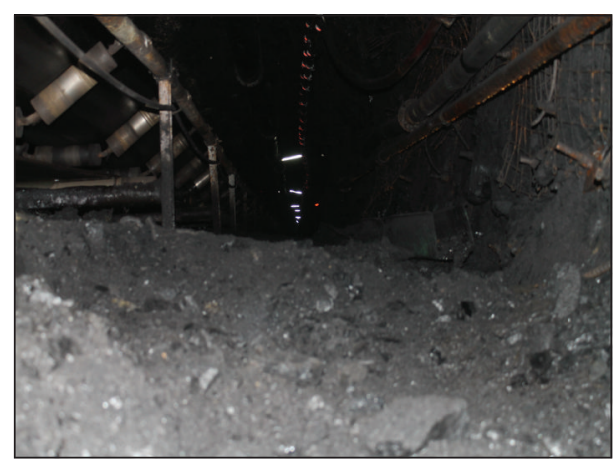

(b) Floor heave

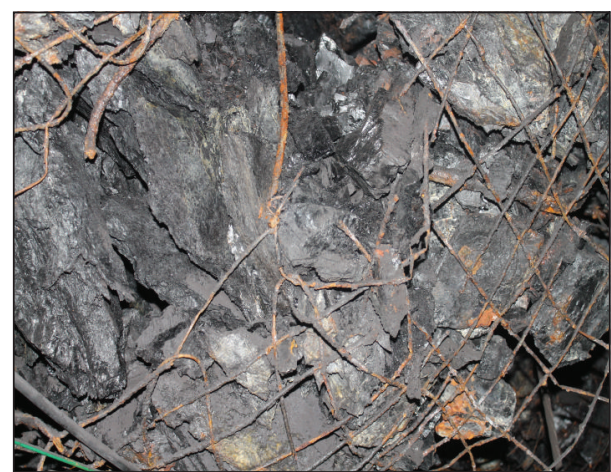

(d) Anchor net tearing

FIGURE 8: Description of destroying phenomena of the 9.18 .

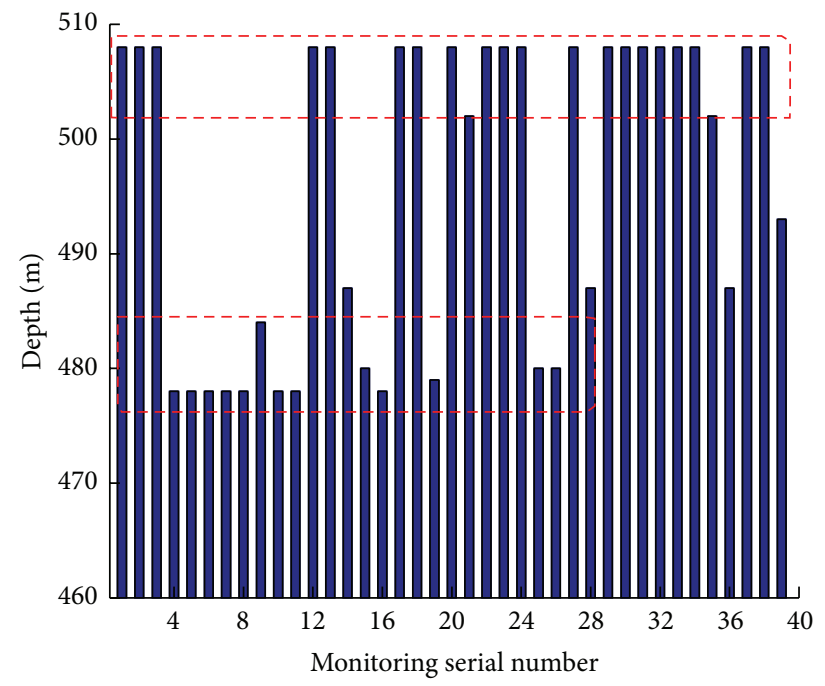

(a) $B_{1-2}$ coal seams

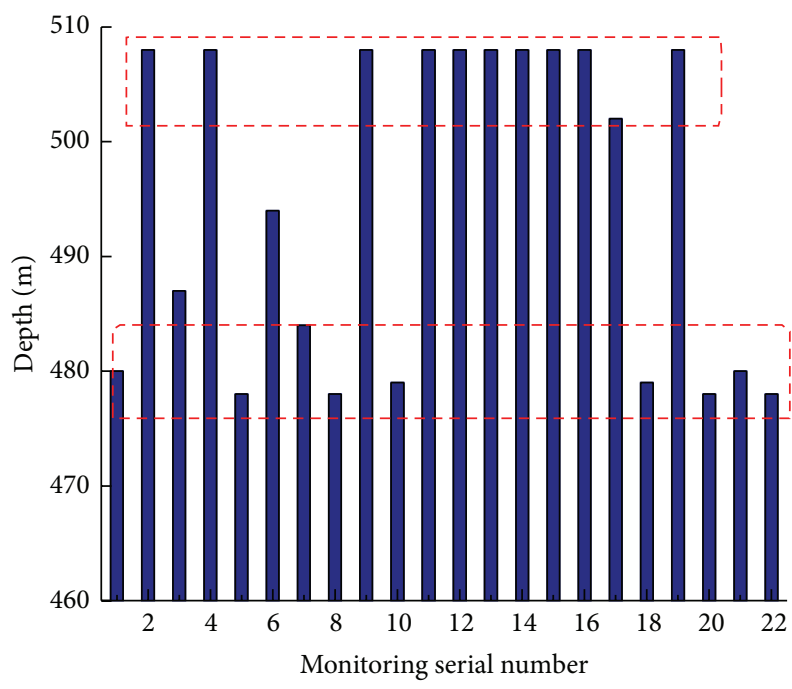

(b) $B_{3-6}$ coal seams

FIGURE 9: Distribution of mining seismicity (2013.9.8 9.22).

$350 \mathrm{~m}$. Deeper mining would result in high stress concentration so as to induce mining seismicity. In the unique geological and unwatered environment of the steep-heavy-thick coal, extraction of the coal seam in subhorizontal section would meet the multioverlying strata and the mined-out area which consists of a number of barrier pillars with high stress concentrations at the depth of $350 \mathrm{~m}$. The existence of these stressed pillars over the mining actives at deeper bed also becomes a contributing factor to the mining seismicity [18]. In general, the mining seismicity that happened in Urumchi coalfield is quite unique because it is rooted in a narrow mining space. 
5.2. Coupling of Spatial-Temporal Strength Characteristics and Redisturbance. Indubitably, underground mining disturbance is a nonlinear dynamic evolvement process related to the spatial-temporal-strength, and the stress concentration of the top-coal and rock masses is caused by the mobilization effect of the arch-pry and redisturbance from deep and complex structures in steeply dipping thick coal seams. Under the action of high stress concentration, the scale of the near field fractured zone sequentially migrates to the deeper roof of the roadway, which caused the aggregation of multiple fracture zones with a poor stability at the combination of the vertical wall and arch. It indicates that mining seismicity gestation and occurrence are derived from multi-index coupling contribution patterns. Moreover, different depth and scope of fractured zones are formed in the far field stress released area during stress migration. Finally, spatial-temporal-strength evolution of the mining disturbed energy induces mining seismicity, coal-rock mass burst, and serious dynamic hazard.

5.3. Stress-Lever-Rotation-Effect (SLRE). According to the horizon of the coal seam thickness, the working face is arranged with a short length during the top-coal caving in horizontal section. However, no roof but top-coal and rock residues are reestablished above the working face during shallow mining. Instability of the arch of spanning strata (ASS) results in the top-coal sliding and structure instability.

After mining the deeper coal-seam and disturbing the rock pillar, the root segments of the steeply deeper rock pillar adjacent to both lower and upper working faces or headings were nonuniformly mobilized; then the effects of heterogeneity on equivalent modulus and failure were manifested by the upper additional dynamic load that stemmed from the upper broken strata. Accordingly, the stress-lever-rotationeffect (SLRE) of the fault-like mobilization would induce and even intensify dynamic hazard [19]. It is also applicable for the arch of spanning strata (ASS) in vertically inhomogeneous anisotropic rock masses.

5.4. Validity of Field Monitoring Index. On the basis of field experiences, it is observed that these advanced monitoring instruments to be preferably applied in and around SSTCC workings (coal, heading, and pillar) are less dependent upon the geomining conditions of the sites. Acoustic-wave and borehole imaging monitoring verifies the asymmetric characteristics of deformation and fracture distribution. The electromagnetic wave detection shows that broken strata will absorb large amounts of energy of electromagnetic wave. It would provide essential data to assess both dynamic hazard prevention and deep mining potential.

Above all, acoustic-seismic-wave index indicated the spatial-temporal-strength and mining disturbance and stress redistribution of the steep-heavy-thick coal and rock masses. The prediction and evaluation for mining seismicity are applicable and valid obviously. The shallow-focus earthquake (6.6 Ms) and aftershock happened on June 30, 2012. The advance heading of number 45 coal seam working face induced severe tremors and bolt-cables broken and a section of metal mesh located between $756.7 \mathrm{~m}$ and $762.0 \mathrm{~m}$ was split; about 21 tons of coal was burst out. Fortunately it is none of mortalities. It is causal of previously applied methods of hydraulic fractured and preblasting, mining optimization, pillar reinforcement, and depressed stress along dipping angles. Operating practice shows that the environment, safety, and productions have been improved. The highest efficiency was $77 \mathrm{t} / \mathrm{d}$, the average efficiency was $60.84 \mathrm{t} / \mathrm{d}$, which was increased by $35.2 \%$, the recovery rate was $85.21 \%$, and the highest production was $6000 \mathrm{t} / \mathrm{d}$. Gas concentration was below $0.1 \%$, and dust concentration was reduced by $10.0 \%$ and hydrogen sulfide concentration was significantly reduced, all of which improve the environment better and reduce the labor intensity of workers.

\section{Conclusions}

(1) Mining seismicity is a typical dynamic hazard in high ground stress region of western China. Innovation of field monitoring is a crucial way to predict mining seismicity.

(2) The stress-lever-rotation-effect (SLRE) model of faultlike mobilization is preliminarily proposed. Based on the effect of the arch of spanning strata (ASS) and predisturbance, the crack distribution, and broken presented asymmetrical migration and localization, steeply deeper rock mass will induce stress concentration obviously when the fracture of the coal is larger. The stress-lever-effect of deeper rock pillar will induce dynamic hazard. It is also applicable for the vertically inhomogeneous anisotropic rock masses.

(3) The characteristics of acoustic-seismic-wave can indicate the spatial-temporal-strength, redisturbance, and stress redistribution of steeply deeper-heavy thick coal and rock masses. The prediction and evaluation for mining seismicity are applicable and valid.

\section{Conflict of Interests}

The authors declare that there is no conflict of interests regarding the publication of this paper.

\section{Acknowledgments}

Financial support for this work provided by 973 National Key Basic Research Development program (2015CB251600), 973 Preliminary National Key Basic Research Development program (2014CB260404), the Key National Natural Science Foundation of China (U13612030), Shaanxi Innovation Team Program (2013KCT-16), and the High Technology Development Program of Xinjiang Municipality (201432102) is gratefully acknowledged.

\section{References}

[1] M. F. Cai, H. G. Ji, and J. A. Wang, "Study of the time-spacestrength relation for mining seismicity at Laohutai coal mine and its prediction," International Journal of Rock Mechanics and Mining Sciences, vol. 42, no. 1, pp. 145-151, 2005.

[2] X.-P. Lai, N.-B. Wang, H.-D. Xu, T. Qi, J.-T. Cao, and D.-H. Jiang, "Safety top-coal-caving of heavy and steep coal seams under 
complex environment," Journal of University of Science and Technology Beijing, vol. 31, no. 3, pp. 277-280, 2009 (Chinese).

[3] X.-P. Lai, F.-H. Ren, Y.-P. Wu, and M.-F. Cai, "Comprehensive assessment on dynamic roof instability under fractured rock mass conditions in the excavation disturbed zone," International Journal of Minerals, Metallurgy and Materials, vol. 16, no. 1, pp. 12-18, 2009.

[4] S.-J. Miao, X.-P. Lai, X.-G. Zhao, and F.-H. Ren, "Simulation experiment of AE-based localization damage and deformation characteristic on covering rock in mined-out area," International Journal of Minerals, Metallurgy and Materials, vol. 16, no. 3, pp. 255-260, 2009.

[5] X. Lai, P. Shan, J. Cao, H. Sun, Z. Suo, and F. Cui, "Hybrid assessment of pre-blasting weakening to horizontal section top coal caving (HSTCC) in steep and thick seams," International Journal of Mining Science and Technology, vol. 24, no. 1, pp. 3137, 2014.

[6] J.-C. Wang, J.-L. Yang, H.-H. Liu, D.-P. Zhao, and L.-Y. Zheng, "The practical observation research on loose medium flow field theory on the top-coal caving," Journal of the China Coal Society, vol. 35, no. 3, pp. 353-356, 2010 (Chinese).

[7] H. Alehossein and B. A. Poulsen, "Stress analysis of longwall top coal caving," International Journal of Rock Mechanics and Mining Sciences, vol. 47, no. 1, pp. 30-41, 2010.

[8] S. Karekal, R. Das, L. Mosse, and P. W. Cleary, "Application of a mesh-free continuum method for simulation of rock caving processes," International Journal of Rock Mechanics and Mining Sciences, vol. 48, no. 5, pp. 703-711, 2011.

[9] Y.-S. Xie and Y.-S. Zhao, "Numerical simulation of the top coal caving process using the discrete element method," International Journal of Rock Mechanics and Mining Sciences, vol. 46, no. 6, pp. 983-991, 2009.

[10] F. Simsir and M. K. Ozfirat, "Determination of the most effective longwall equipment combination in longwall top coal caving (LTCC) method by simulation modelling," International Journal of Rock Mechanics and Mining Sciences, vol. 45, no. 6, pp. 1015-1023, 2008.

[11] C.-P. Lu and L.-M. Dou, "The relationship between vertical stress gradient, seismic, and electromagnetic emission signals at Sanhejian coal mine, China," International Journal of Rock Mechanics and Mining Sciences, vol. 70, pp. 90-100, 2014.

[12] P.-W. Shi and Y.-Z. Zhang, "Structural analysis of arch of spanning strata of top coal caving in steep seam," Chinese Journal of Rock Mechanics and Engineering, vol. 25, no. 1, pp. 79-82, 2006.

[13] L. Xingping, W. Yongping, C. Jiantao et al., The Foundmental Research on Mechanics and Engineering of Safe and High Efficiency Mining for the Steeply Deeper Heavy-Thick Coal Seams, Shan-xi Science Press, Xi'an, China, 2013.

[14] X.-P. Lai, Y.-P. Wu, J.-T. Cao, Y.-N. Fan, Y.-L. Zhang, and F. Cui, "Experiment on rock-mass deformation of large scale 3Dsimulation in complex environment," Journal of the China Coal Society, vol. 35, no. 1, pp. 31-36, 2010 (Chinese).

[15] C.-P. Lu, L.-M. Dou, N. Zhang et al., "Microseismic frequencyspectrum evolutionary rule of rockburst triggered by roof fall," International Journal of Rock Mechanics and Mining Sciences, vol. 64, pp. 6-16, 2013.

[16] C. P. Lu, L. M. Dou, H. Liu, H. S. Liu, B. Liu, and B. B. Du, "Case study on microseismic effect of coal and gas outburst process," International Journal of Rock Mechanics and Mining Sciences, vol. 53, pp. 101-110, 2012.

[17] C.-P. Lu, L.-M. Dou, X.-R. Wu, and Y.-S. Xie, "Case study of blast-induced shock wave propagation in coal and rock,"
International Journal of Rock Mechanics and Mining Sciences, vol. 47, no. 6, pp. 1046-1054, 2010.

[18] C. Feng, Fundamental research on the coupled-fracture and application for coal-rock under complex environment [Ph.D. dissertation], Xi'an University of Science and Technology, 2014.

[19] J. Cao, Prediction and control research on mining seismicity occurrences under complex coal-rock masses [Ph.D. dissertation], Xi'an University of Science and Technology, 2014. 

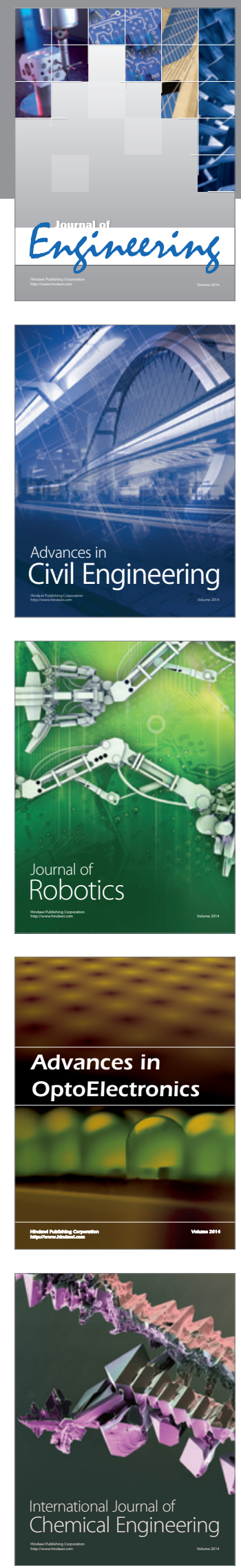

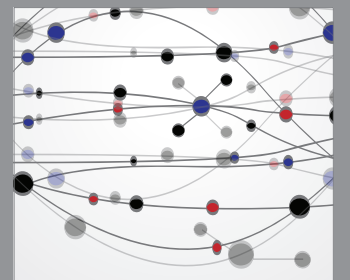

The Scientific World Journal
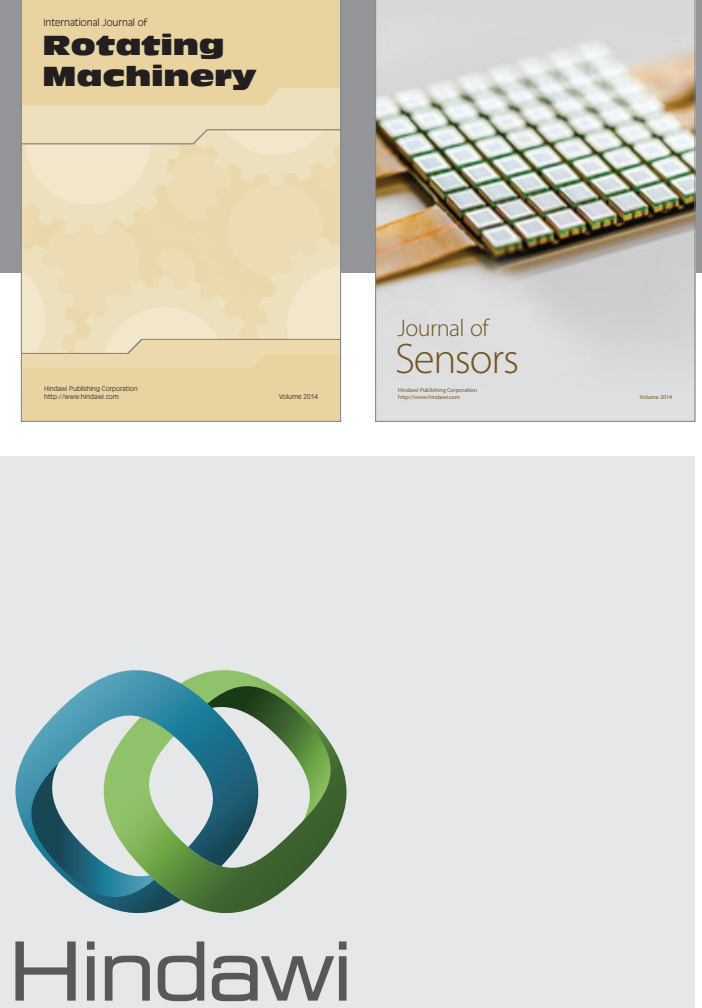

Submit your manuscripts at http://www.hindawi.com
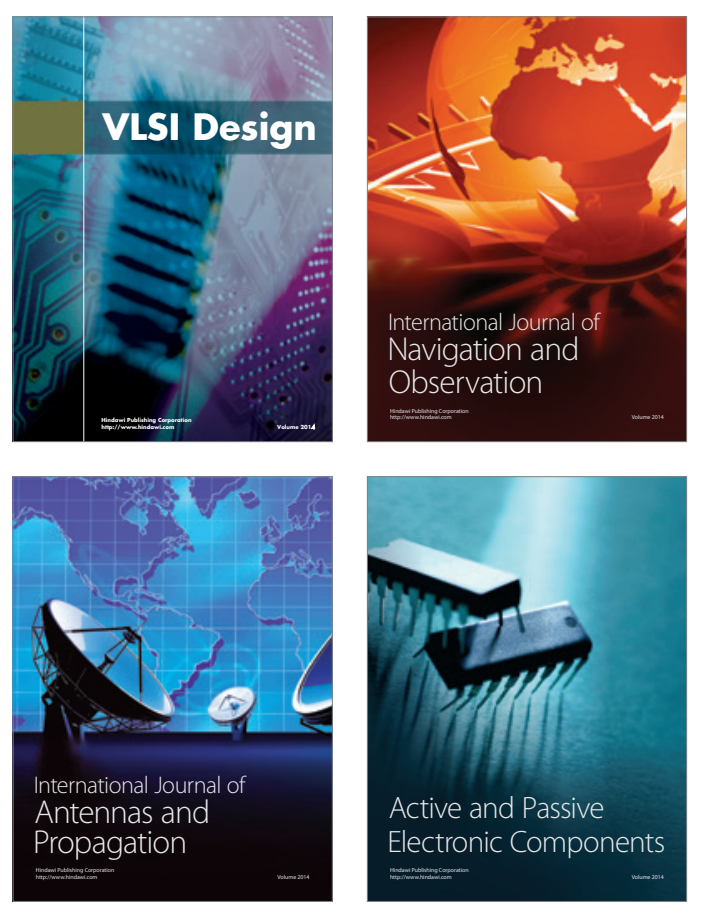
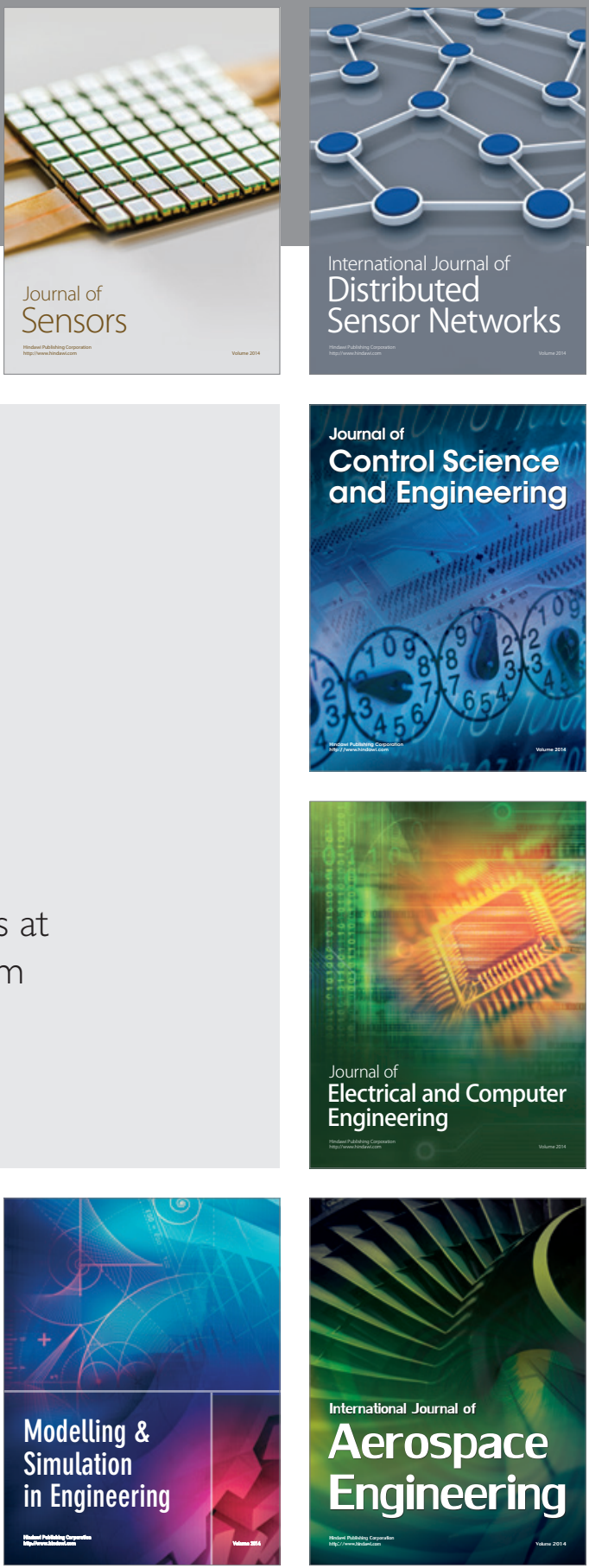

Journal of

Control Science

and Engineering
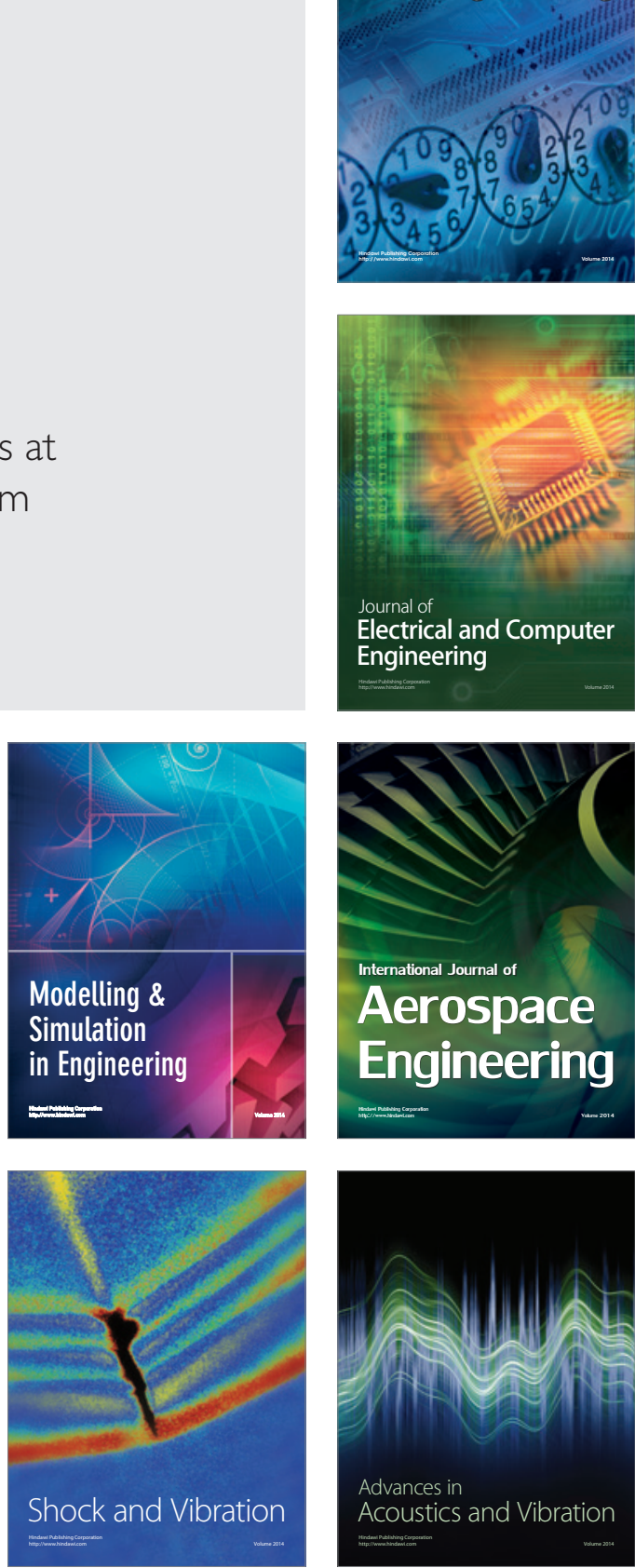